UDC 530.12

DOI: $10.22363 / 2312-9735-2017-25-4-390-400$

\title{
Two Approaches to Interpretation of Hubble Diagram
}

\author{
A. E. Pavlov
}

\author{
Department of Strength of Materials and Machine Parts \\ Russian State Agrarian University - Moscow Timiryazev Agricultural Academy \\ 49 Timiryazevskaya St., Moscow, 127550, Russian Federation
}

\begin{abstract}
Supernovae of type Ia are used as standard candles in modern cosmology, they serve to test cosmological models. Interpretation of the Hubble diagram based on the standard cosmological model led cosmologists to conclusion that the Universe is filled mostly with cosmic dust and mysterious dark energy.

In this paper we present exact solutions of the Friedmann equation in standard cosmology and conformal cosmology. The theoretical curves interpolating the Hubble diagram for the latest supernova data are expressed in an analytical form. The functions belong to the class of meromorphic Weierstrass functions. Both approaches describe the modern Hubble diagram with the same accuracy. Physical interpretation from the standpoint of conformal cosmology is preferable, since supernova data are described without using a $\Lambda$-term. In the standard cosmology, the Hubble diagram is described by some characteristics: a Hubble parameter $H(t)$, a deceleration $q(t)$, and a jerk $j(t)$. As calculations show, the deceleration parameter $q$ changes its sign during the evolution of the Universe, the $j$-parameter remains constant. In the modern era, the Universe expands with acceleration, and in the past its acceleration was negative. The change in the sign of acceleration, without a clear physical reason, puzzles cosmologists.

It seems obvious to us that to study objects dislocated from us at distances of billions of light years, we should not use the coordinate time customary for work in laboratories, but the conformal time. In conformal coordinates, the behavior of photons is described as in the Minkowski space. The time intervals $\mathrm{d} t$ and $\mathrm{d} \eta$ are different, they are related by the scale factor: $\mathrm{d} t=a \mathrm{~d} \eta$. The conformal luminosity distance is longer than the standard luminosity distance, which is manifested when observing distant stellar objects. As a result, the effective magnitude value - the redshift relationship, on which the Hubble diagram is constructed, will be different. Using the conformal Friedmann equation, we introduce the conformal parameters $H(\eta), q(\eta)$, $j(\eta)$. All parameters remain positive during the evolution of the Universe. The scale factor grows with deceleration. The Universe does not experience a jerk.
\end{abstract}

Key words and phrases: supernovae of type Ia, Friedmann equation, Weierstrass functions, Hubble diagram, cosmological parameters, $\Lambda$-term

\section{Introduction}

A type Ia supernova occurs when a white dwarf in a double star system accumulates a mass by accretion that sufficient to overcome the Chandrasekhar limit. The nature of explosion of such a supernova depends on its prehistory insignificantly. Redshifts $z>0.1$ are large enough to ignore peculiar motions of light sources. Supernovae type Ia, sufficiently bright stars whose absolute luminosity is known with good accuracy, they serve as standard candles for testing cosmological models. Two collaborations The Supernova Cosmology Project and High-z Supernova Search Team compared the results of observations of supernovae with theoretical predictions for luminosity distances as redshift functions. Interpretation of the Hubble diagram on the basis of a standard cosmological model with adjustable cosmological parameters led cosmologists to conclusion that the Universe is filled mainly with dust and, so-called, dark energy - a substance with an equation of state not found in Nature [1-3]. Phenomenological approach has not led to an understanding of the state of matter from which the Universe consists.

Conformal cosmological model [4], based on conformal Dirac's variables [5], allows to explain data on supernovae without $\Lambda$-term [6-9]. We show that solutions of

Received $25^{\text {th }}$ April, 2017. 
the differential Friedmann equation belong to the class of Weierstrass meromorphic functions [10]. Therefore, it is natural to use them to compare predictions of these two approaches [11-13]. In conclusion, we show that the difference between distance modulus is interpolated by means of the Chebyshev polynomial of the fourth order with a sufficient degree of accuracy.

\section{Friedmann equation in classical cosmology}

In the standard cosmological model the Friedmann equation

$$
\left(\frac{\dot{a}}{a}\right)^{2}=\frac{8 \pi G}{3} \rho-\frac{k}{a^{2}}
$$

is used for fitting SNe Ia data. It ties a scale of the Universe $a(t)$ with density of matter $\rho$. Here $G$ is Newton's constant, $k$ is a sign of curvature of a space, a dot denotes a derivative with respect to coordinate time $t$. In generic case, the equation (1) is represented in the following form

$$
\frac{1}{x^{2}}\left(\frac{\mathrm{d} x}{\mathrm{~d} t}\right)^{2}=H_{0}^{2}\left[\Omega_{\Lambda}+\Omega_{\mathrm{curv}} x^{-2}+\Omega_{\mathrm{M}} x^{-3}+\Omega_{\mathrm{rad}} x^{-4}+\Omega_{\mathrm{rigid}} x^{-6}\right],
$$

where the variable $x$ is given as a ratio of a scale $a(t)$ to a modern one $a_{0}=1$ :

$$
x \equiv \frac{a(t)}{a_{0}}=\frac{1}{1+z}
$$

$z$ is a redshift of spectral lines (observed variable), $H_{0}=h \cdot 10^{5} \mathrm{~m} / \mathrm{s} / \mathrm{Mpc}, h=0.72 \pm 0.08-$ Hubble constant. In the right hand of the Friedmann equation (2) $\Omega_{i}$ are partial densities of, correspondingly, $\Lambda$-term, curvature of the space, dust-like matter, radiation, stiff-state matter. For distant sources with $z>1$ the interpretation of the cosmological redshift as a Doppler shift is not valid [14]. An equation of continuity

$$
\dot{\rho}=-\frac{3 \dot{a}}{a}(\rho+p),
$$

with an equation of state of matter $p=w \rho$, which connects the density $\rho$ and the pressure $p$, yields the dependence of the density on the scale factor. So,

- for interstellar dust $p=0: \rho \sim a^{-3}$;

- for radiation $p=\rho / 3: \rho \sim a^{-4}$;

- for contribution from $\Lambda$-term $p=-\rho: \rho \sim \Lambda$;

- for stiff state of matter $p=\rho: \rho \sim a^{-6}$.

The data of modern astronomical observations are fitted using cosmological parameters [2]: $\Omega_{\Lambda}=0.72, \Omega_{\mathrm{M}}=0.28$. A solution of the Friedmann equation (2) with such parameters is presented in analytical form

$$
\left.a(t)=a_{0} \sqrt[3]{\frac{\Omega_{\mathrm{M}}}{\Omega_{\Lambda}}}\left[\operatorname{sh}\left(\frac{3}{2} \sqrt{\Omega_{\Lambda}} H_{0} t\right)\right)\right]^{2 / 3} .
$$

The second derivative of the scale factor is

$$
\ddot{a}=\frac{H_{0}^{2} a_{0}}{2}\left[2 \Omega_{\Lambda}\left(\frac{a}{a_{0}}\right)-\Omega_{\mathrm{M}}\left(\frac{a_{0}}{a}\right)^{2}\right] .
$$


Hence, in the modern era the Universe expands with acceleration, since $2 \Omega_{\Lambda}>\Omega_{\mathrm{M}}$; in the past, its acceleration was negative $\ddot{a}<0$. This change of the sign of acceleration without a clear physical cause is noted by cosmologists. From the solution (4), taking into account the relation between the scale factor and the redshift (3), it follows age redshift relationship

$$
H_{0} t=\frac{2}{3 \sqrt{\Omega_{\Lambda}}} \operatorname{Arsh}\left(\sqrt{\frac{\Omega_{\Lambda}}{\Omega_{M}}} \frac{1}{(1+z)^{3 / 2}}\right)
$$

The age $t_{0}$ of the modern Universe in the coordinate time can be obtained by setting $z=0$ in $(6)$

$$
t_{0}=\frac{2}{3 \sqrt{\Omega_{\Lambda}}} \frac{1}{H_{0}} \operatorname{Arsh} \sqrt{\frac{\Omega_{\Lambda}}{\Omega_{M}}} .
$$

If we know the redshift $z$ of a certain galaxy, how do we find the coordinate distance $r$ to it? Since for the rays of light the spacetime interval is zero

$$
\mathrm{d} s^{2}=-c^{2} \mathrm{~d} t^{2}+a^{2}(t) \mathrm{d} r^{2}=0
$$

we have the relation between intervals of space and time $c \mathrm{~d} t=-a(t) \mathrm{d} r$, and, using the notation introduced above $(3) x \equiv a / a_{0}$,

$$
-a_{0} r=c \int \frac{\mathrm{d} t}{x}=c \int \frac{\mathrm{d} x}{x} \frac{1}{\mathrm{~d} x / \mathrm{d} t} .
$$

Substituting now the Friedmann equation (2) into (8)

$$
\frac{\mathrm{d} x}{\mathrm{~d} t}=H_{0} \sqrt{\Omega_{\mathrm{M}} / x+\Omega_{\Lambda} x^{2}},
$$

we get an integral

$$
H_{0} r=\frac{c}{\sqrt{\Omega_{\Lambda}}} \int_{1 /(1+z)}^{1} \frac{\mathrm{d} x}{\sqrt{x^{4}+4 a_{3} x}},
$$

where we denote the ratio of partial densities as $4 a_{3} \equiv \Omega_{\mathrm{M}} / \Omega_{\Lambda}$. The integral is calculated using the inverse Weierstrass $\wp$-function [12] and one obtains coordinate distance redshift relationship

$$
H_{0} r=-\frac{c}{\sqrt{\Omega_{\Lambda}}} \wp^{-1}\left[\frac{1-\sqrt{1+\Omega_{\mathrm{M}} / \Omega_{\Lambda}(1+z)^{3}}}{2(1+z)^{2}}\right]+\frac{c}{\sqrt{\Omega_{\Lambda}}} \wp^{-1}\left[\frac{\left(1-\sqrt{1+\Omega_{\mathrm{M}} / \Omega_{\Lambda}}\right)}{2}\right] .
$$

The resulting formula is expressed in terms of the Weierstrass $\wp$-function [10], satisfying the differential equation

$$
\left[\frac{d \wp(u)}{d u}\right]^{2}=4\left[\wp(u)-e_{1}\right]\left[\wp(u)-e_{2}\right]\left[\wp(u)-e_{3}\right]
$$


where $\wp\left(\omega_{\alpha}\right)=e_{\alpha}, \wp^{\prime}\left(\omega_{\alpha}\right)=0, \alpha=1,2,3$ - three roots of a cubic polynomial on the right-hand side of the differential equation (12)

$$
e_{1} \equiv \frac{1}{8}\left(\frac{\Omega_{\mathrm{M}}}{\Omega_{\Lambda}}\right)^{2 / 3}(1+\imath \sqrt{3}), \quad e_{2} \equiv-\frac{1}{4}\left(\frac{\Omega_{\mathrm{M}}}{\Omega_{\Lambda}}\right)^{2 / 3}, \quad e_{3} \equiv \frac{1}{8}\left(\frac{\Omega_{\mathrm{M}}}{\Omega_{\Lambda}}\right)^{2 / 3}(1-\imath \sqrt{3}) .
$$

Invariants of the Weierstrass function are the following

$$
g_{2}=0, \quad g_{3}=-a_{3}^{2}=-\left(\frac{\Omega_{\mathrm{M}}}{4 \Omega_{\Lambda}}\right)^{2} ;
$$

the discriminant of a cubic polynomial is negative: $\Delta \equiv g_{2}^{3}-27 g_{3}^{2}<0$.

In astronomy, the method of determining distances to ultra-distant objects is based on measuring their luminosity. The radiation power $L$ of an object (a star or a galaxy) is called its absolute luminosity. The flux density $\ell$, i.e., the radiation power per unit area, is called its visible luminosity. In Euclidean geometry they are connected by the formula

$$
\ell=\frac{L}{4 \pi d^{2}},
$$

where $d$ is the distance from us to the radiation object. In the second century BC the Greek astronomer Hipparchus classified the stars visible to the naked eye into six classes according to their brightness. The brightest stars were assigned the first magnitude, and barely visible - the sixth magnitude. According to Norman Pogson in 1856 it was decided that the luminosity of the objects of the first stellar magnitude is a hundred times greater than the luminosity of the objects of the sixth stellar magnitude [14], i.e.,

$$
\ell=\ell_{0} \cdot 100^{-m / 5}, \quad L=L_{0} \cdot 100^{-M / 5},
$$

where $\ell_{0}$ and $L_{0}$ are some relevant luminosities. With the creation of photomultipliers at the beginning of the XX-th century, the factors $\ell_{0}$ and $L_{0}$ were fixed. We express from (14) the apparent stellar magnitude $m$ and the absolute stellar magnitude of the object $M$ using the decimal logarithms

$$
m=-\frac{5}{2} \lg \frac{\ell}{\ell_{0}}, \quad M=-\frac{5}{2} \lg \frac{L}{L_{0}} .
$$

Then we express from (15) the distance modulus $(m-M)$ through the distance $d$ to the radiation object, using (13)

$$
m-M=-\frac{5}{2} \lg \left(\frac{\ell}{L} \cdot \frac{L_{0}}{\ell_{0}}\right)=\frac{5}{2} \lg \left(4 \pi d^{2} \cdot \frac{L_{0}}{\ell_{0}}\right)=5 \lg d+\frac{5}{2} \lg \left(4 \pi \frac{\ell_{0}}{L_{0}}\right) .
$$

For performing calculations, the factors $\ell_{0}$ and $L_{0}$ in (14) are chosen in such a way that the distance $d$ is measured in megaparsecs

$$
m-M=5 \lg d(\mathrm{Mpc})+\mathcal{M},
$$

where $\mathcal{M}=25$.

In the Friedman-Robertson-Walker cosmology, by analogy with the formula for the distance $d$ in Euclidean geometry (13), we determine a luminosity distance $d_{L}$ to a star object

$$
d_{L} \equiv \sqrt{\frac{L}{4 \pi \ell}}
$$


In standard cosmology, the luminosity distance $d_{L}(z)_{S C}$ is related to the coordinate distance $r$ [2]:

$$
d_{L}(z)_{S C}=(1+z) a_{0} r .
$$

Here it was taken into account that the area of the sphere around the luminous object passing through the Earth increases, the frequency of photons decreases during their motion. Substituting the formula for the coordinate distance (11) into (18), we get an analytical expression for luminosity distance

$$
d_{L}(z)_{S C}=\frac{c(1+z)}{H_{0} \sqrt{\Omega_{\Lambda}}}\left(\wp^{-1}\left[\frac{\left(1-\sqrt{1+\Omega_{\mathrm{M}} / \Omega_{\Lambda}}\right)}{2}\right]-\wp^{-1}\left[\frac{1-\sqrt{1+\Omega_{\mathrm{M}} / \Omega_{\Lambda}(1+z)^{3}}}{2(1+z)^{2}}\right]\right) .
$$

Modern observational cosmology is based on the Hubble diagram. Effective stellar magnitude - redshift relationship

$$
m(z)-M=5 \lg \left[d_{L}(z)_{S C}\right]+\mathcal{M},
$$

is used to test cosmological theories $\left(d_{L}\right.$ in megaparsecs) [2]. Here $m(z)$ is the apparent magnitude, $M$ is its absolute magnitude, and $\mathcal{M}=25$ is a constant.

\section{Friedmann equation in conformal cosmology}

Interpretation of the Hubble diagram, based on a conformal cosmological model with parameters $\Omega_{\text {rigid }}=0.755, \Omega_{\mathrm{M}}=0.245$, yields the same qualitative approximation as the standard cosmological model with parameters $\Omega_{\Lambda}=0.72, \Omega_{\mathrm{M}}=0.28$ [9]. A parameter $\Omega_{\text {rigid }}$ corresponds to a stiff state of matter, when the energy density is equal to the pressure $p=\rho[15,16]$ that is happened under a nucleosynthesis regime in stars. We write out conformal Friedmann equation [4] with using meaningful conformal partial parameters, discarding insignificant contributions

$$
\left(\frac{a^{\prime}}{a}\right)^{2}=\left(\frac{\mathcal{H}_{0}}{c}\right)^{2}\left[\Omega_{\text {rigid }}\left(\frac{a_{0}}{a}\right)^{4}+\Omega_{\mathrm{M}}\left(\frac{a_{0}}{a}\right)\right] .
$$

The right-hand side of the equation (20) includes the densities of the state of matter $\rho(a)$ in accordance with their conformal weights; in the left, the prime denotes the derivative with respect to the conformal time. After introducing the dimensionless variable $x \equiv a / a_{0}$, the conformal Friedmann equation (20) takes the form

$$
\left(\frac{2 c}{\sqrt{\Omega_{\mathrm{M}}} \mathcal{H}_{0}}\right)^{2} x^{2}\left(\frac{\mathrm{d} x}{\mathrm{~d} \eta}\right)^{2}=4 x^{3}-g_{3} \equiv 4\left(x-e_{1}\right)\left(x-e_{2}\right)\left(x-e_{3}\right),
$$

where one root in the cubic polynomial on the right-hand side (21) is real, while the other ones are complex conjugated

$$
e_{1} \equiv \sqrt[3]{\frac{\Omega_{\text {rigid }}}{\Omega_{\mathrm{M}}}} \frac{1+\imath \sqrt{3}}{2}, \quad e_{2} \equiv-\sqrt[3]{\frac{\Omega_{\text {rigid }}}{\Omega_{\mathrm{M}}}}, \quad e_{3} \equiv \sqrt[3]{\frac{\Omega_{\text {rigid }}}{\Omega_{\mathrm{M}}}} \frac{1-\imath \sqrt{3}}{2} .
$$

The invariants of the cubic polynomial are as following:

$$
g_{2}=0, \quad g_{3}=-\frac{4 \Omega_{\text {rigid }}}{\Omega_{\mathrm{M}}} .
$$


where $\mathcal{H}_{0}$ is a conformal Hubble constant. The conformal Hubble parameter is related to the usual parameter as $\mathcal{H} \equiv\left(a / a_{0}\right) H$. Then we introduce a new variable $u$ by the rule

$$
x=\wp(u)=-\frac{d \zeta(u)}{u} .
$$

The Weierstrass $\zeta$-function satisfies the quasi-periodicity conditions [10]

$$
\zeta(u+2 \omega)=\zeta(u)+2 \zeta(\omega), \quad \zeta\left(u+2 \omega^{\prime}\right)=\zeta(u)+2 \zeta\left(\omega^{\prime}\right),
$$

where $\omega$ and $\omega^{\prime}$ are semi-periods of the function $\wp(u)$.

The conformal Friedmann equation (21) is integrated and we obtain the conformal age - redshift relationship in explicit form

$$
\eta=\frac{2 c}{\sqrt{\Omega_{\mathrm{M}}} \mathcal{H}_{0}}\left(\zeta\left[\wp^{-1}\left(\frac{1}{1+z}\right)\right]-\zeta\left[\wp^{-1}(1)\right]\right) .
$$

The equation written out in integral form is known in cosmology as the Hubble law. An explicit formula for conformal age of the Universe $\eta_{0}$ can be obtained by putting in (23) $z=0$ :

$$
\eta_{0}=\frac{2 c}{\sqrt{\Omega_{\mathrm{M}}} \mathcal{H}_{0}}\left(\zeta\left[\wp^{-1}(0)\right]-\zeta\left[\wp^{-1}(1)\right]\right) .
$$

The interval of the coordinate conformal distance is equal to the interval of the conformal time $\mathrm{d} r=\mathrm{d} \eta$, so we can represent (23) as the conformal distance - redshift relationship.

In conformal coordinates, the behavior of photons is exactly the same as in the Minkowski space. The time intervals between the emission of two photons and between their absorption are the same. The time interval $\mathrm{d} t=-a \mathrm{~d} r$, used in standard cosmology, and the time interval $\mathrm{d} \eta=-\mathrm{d} r$, used in conformal cosmology, are different. The conformal luminosity distance $d_{L}(z)_{C C}$ is related to the standard luminosity distance $d_{L}(z)_{S C}[9]$

$$
d_{L}(z)_{C C}=(1+z) d_{L}(z)_{S C}=(1+z)^{2} r(z),
$$

where $r(z)$ is a coordinate distance. For photons $\mathrm{d} r / d \eta=-1$, so we get an explicit dependence: luminosity distance - redshift relationship

$$
d_{L}(z)_{C C}=\frac{2 c(1+z)^{2}}{\sqrt{\Omega_{\mathrm{M}}} \mathcal{H}_{0}}\left(\zeta\left[\wp^{-1}\left(\frac{1}{1+z}\right)\right]-\zeta\left[\wp^{-1}(1)\right]\right) .
$$
form

The effective magnitude - redshift relationship in the conformal cosmology has the

$$
m(z)-M=5 \lg \left[d_{L}(z)_{C C}\right]+\mathcal{M} .
$$

\section{Comparative analysis}

According to the conformal cosmological model, the conformal quantities are physical observables. Pearson's criterion $\chi^{2}$ was applied in [9] for statistical fitting of results on supernovae Ia [2]. The contribution from the component corresponding to the rigid state of matter $\rho_{\text {rigid }}$ in the conformal model replaced the contribution from the $\Lambda$-term in the standard model. In the rigid state of matter, its energy density is equal to the pressure. As a result of the analysis, the conclusion was drawn: the best fitting of the conformal model was not inferior to the best fitting of the standard model [9].

The curves for the two models, according to (26) and (19), are shown in Fig. 1. 


\section{$\mathrm{m}-\mathrm{M}$}

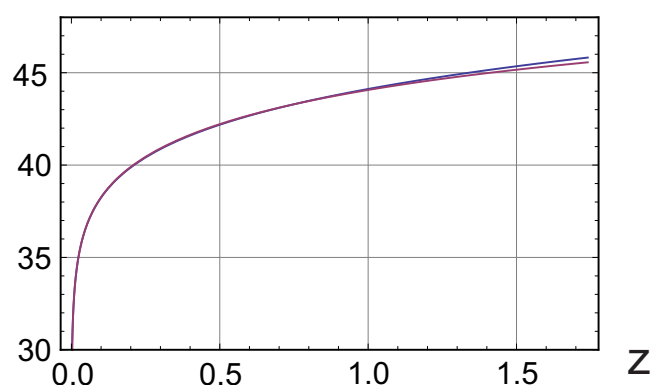

Figure 1. Curves: effective stellar magnitude - redshift for cosmological models considered

The difference between the curves: effective magnitude value - redshift

$$
\Delta(m(z)-M)=5 \lg \left[d_{L}(z)_{C C}\right]-5 \lg \left[d_{L}(z)_{S C}\right]
$$

- predictions of models (26) and (19), is demonstrated in Fig. 2. A slight difference between the curves, within the error of observations, is manifested in the early and late stages of the evolution of the Universe [12].

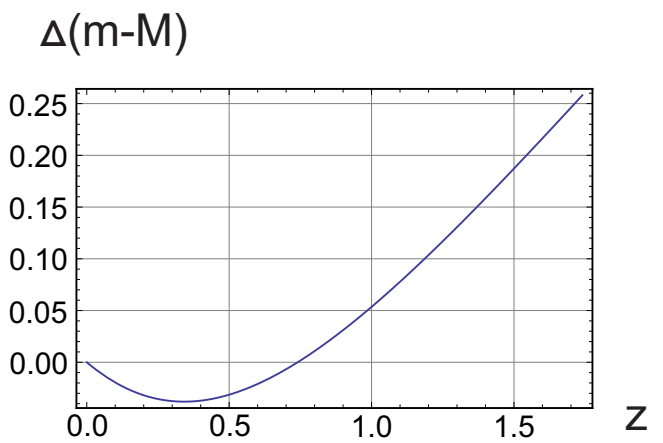

Figure 2. Difference between curves: effective magnitude - redshift for the two models

The function (27) has a complex form, but it is easy interpolated, with a sufficient degree of accuracy, Chebyshev polynomials of the first kind, the least deviating from zero on the interval $[-1 ; 1]$

$$
\Delta(m-M)=\frac{a_{0}}{2}+a_{1} T_{1}(x)+a_{2} T_{2}(x)+a_{3} T_{3}(x)+a_{4} T(x) .
$$

Here you have utilized a variable $-1 \leqslant x \leqslant 1: x \equiv(20 / 17) z-1$, and the coefficients of the expansion have the form: $a_{0} / 2=0.0703, a_{1}=0.0513, a_{2}=0.0513, a_{3}=-0.0123$, $a_{4}=0.0015$. Chebyshev polynomials $[17,18]$ :

$$
T_{0}=1, \quad T_{1}(x)=x, \quad T_{2}(x)=2 x^{2}-1, \quad T_{3}(x)=4 x^{3}-3 x, \quad T_{4}(x)=8 x^{4}-8 x^{2}+1 .
$$


In the standard cosmology, the following characteristics are introduced to describe the Hubble diagram: Hubble parameter, a deceleration and a jerk [2]

$$
\begin{gathered}
H(t) \equiv+\left(\frac{\dot{a}}{a}\right)=H_{0} \sqrt{\frac{\Omega_{\mathrm{M}}}{a^{3}}+\Omega_{\Lambda}}, \\
q(t) \equiv-\left(\frac{\ddot{a}}{a}\right)\left(\frac{\dot{a}}{a}\right)^{-2}=\frac{\Omega_{\mathrm{M}} / 2-\Omega_{\Lambda} a^{3}}{\Omega_{\mathrm{M}}+\Omega_{\Lambda} a^{3}}, \\
j(t) \equiv+\left(\frac{\dot{a}}{a}\right)\left(\frac{\dot{a}}{a}\right)^{-3}=1 .
\end{gathered}
$$

As we see, the deceleration parameter $q(29)$ changes its sign during the evolution of the Universe at the inflection point

$$
a^{*}=\sqrt[3]{\frac{\Omega_{\mathrm{M}}}{2 \Omega_{\Lambda}}},
$$

$j$-parameter remains constant.

We define analogous parameters for the conformal cosmology

$$
\begin{gathered}
\mathcal{H}(\eta) \equiv+\left(\frac{a^{\prime}}{a}\right), \\
q(\eta) \equiv-\left(\frac{a^{\prime \prime}}{a}\right)\left(\frac{a^{\prime}}{a}\right)^{-2}, \\
j(\eta) \equiv+\left(\frac{a^{\prime \prime \prime}}{a}\right)\left(\frac{a^{\prime}}{a}\right)^{-3} .
\end{gathered}
$$

We calculate the conformal parameters using the conformal Friedmann equation (20). Hubble parameter

$$
\mathcal{H}(\eta)=\frac{\mathcal{H}_{0}}{a^{2}} \sqrt{\Omega_{\text {rigid }}+\Omega_{\mathrm{M}} a^{3}}>0 ;
$$

deceleration parameter

$$
q(\eta)=\left(\frac{\Omega_{\text {rigid }}-\left(\Omega_{\mathrm{M}} / 2\right) a^{3}}{\Omega_{\text {rigid }}+\Omega_{\mathrm{M}} a^{3}}\right)>0
$$

changes from 1 to $\left(\Omega_{\text {rigid }}-\Omega_{\mathrm{M}} / 2\right)$; hence, the scale factor grows with deceleration; the jerk parameter

$$
j(\eta)=\frac{3 \Omega_{\text {rigid }}}{\Omega_{\text {rigid }}+\Omega_{\mathrm{M}} a^{3}}>0
$$

changes from 3 to $3 \Omega_{\text {rigid }}$. Dimensionless parameters $q(\eta)$ and $j(\eta)$ remain positive during the evolution. The Universe does not undergo, during its evolution, the so-called jerk artifact of approach of the standard cosmological model.

\section{Conclusions}

We present exact solutions of the Friedmann equation in standard cosmology and conformal cosmology. The theoretical curves interpolating the Hubble diagram for the 
latest supernova data are expressed in an analytical form. The functions belong to the class of meromorphic Weierstrass functions. Both approaches describe the modern Hubble diagram with the same accuracy. We introduce conformal parameters describing the Hubble diagram. All parameters remain positive during the evolution of the Universe.

\section{Acknowledgments}

I am grateful to Professor V.N. Pervushin for discussions on modern theoretical problems that have arisen in cosmology, and comprehensive friendly support, the Laboratory of Theoretical Physics named after N. N. Bogoliubov, Joint Institute for Nuclear Research (Dubna) for hospitality. I express my gratitude for the interest in the work to the participants of the International Winter School "Seminar on gravitation, cosmology and astrophysics" (Petrov readings), Kazan Federal University, participants of the seminar of the Department of Theoretical Physics and Mechanics of the People's Friendship University of Russia (Moscow).

\section{References}

1. A. G. Riess, et al., The Farthest Known Supernova: Support for an Accelerating Universe and a Glimpse of the Epoch of Deceleration, The Astrophysical Journal 560 (2001) 49-71.

2. A. G. Riess, et al., Type Ia Supernova Discoveries at $z>1$ from the Hubble Space Telescope: Evidence for Past Deceleration and Constraints on Dark Energy Evolutions, The Astrophysical Journal 607 (2004) 665-687.

3. A. G. Riess, Nobel Lecture: My Path to the Accelerating Universe, Reviews of Modern Physics 84 (2012) 1165-1175.

4. V. N. Pervushin, A. E. Pavlov, Principles of Quantum Universe, Lambert Academic Publishing, Saarbrücken, 2014.

5. P. A. M. Dirac, Fixation of Coordinates in the Hamiltonian Theory of Gravitation, Physical Review 114 (1959) 924-930.

6. D. Behnke, et al., Description of Supernova Data in Conformal Cosmology without Cosmological Constant, Physics Letters 30 B (2002) 20-26.

7. D. Behnke, Conformal cosmology approach to the problem of dark matter, Ph.D. thesis, PhD Thesis, Rostock Report MPG-VT-UR 248/04 (2004).

8. A. F. Zakharov, V. N. Pervushin, V. A. Zinchuk, Tetrad Formulation and Frames of Reference in General Relativity, Physics of Particles and Nuclei 37 (2006) 104-134.

9. A. F. Zakharov, V. N. Pervushin, Conformal Cosmological Model Parameters with Distant SNe Ia Data: "Gold" and "Silver", International Journal of Modern Physics 19 D (2010) 1875-1886.

10. E. T. Whittaker, G. N. Watson, A Course of Modern Analysis, Cambridge University Press, Cambridge, 1927.

11. A. E. Pavlov, Exact Solutions of Friedmann Equation for Supernovae Data (2015). URL https://arxiv.org/pdf/1511.00226.pdf

12. A. E. Pavlov, Intrinsic Time in Geometrodynamics: Introduction and Application to Friedmann Cosmology (2016). URL https://arxiv.org/pdf/1606.09460

13. A. E. Pavlov, Parameters of the Hubble Diagram, in: 2-nd International Winter School-Seminar on gravity, cosmology, and astrophysics. "Petrov School - 2016", Kazan Federal University, 2016, pp. 29-30.

14. S. Weinberg, Cosmology, Oxford University Press, Oxford, 2008.

15. Ya. B. Zel'dovich, The Equation of State at Ultrahigh Densities and its Relativistic Limitations, Soviet Physics JETP 14 (1962) 1143-1147.

16. J. Narlikar, Violent Phenomena in the Universe, Oxford University Press, Oxford, 1984. 
17. N. I. Akhiezer, Lections on Theory of Approximation, Nauka, Moscow, 1965.

18. N. I. Akhiezer, Elements of Theory of Elliptic Functions, Nauka, Moscow, 1970.

УДК 530.12

DOI: $10.22363 / 2312-9735-2017-25-4-390-400$

\title{
Два подхода к интерпретации диаграммы Хаббла
}

\section{А. Е. Павлов}

\author{
Кафедра сопротивления материалов и деталей машин \\ Российский государственный аграрный университет-MCXА им. К. А. Тимирязева \\ Тимирязевская ул., д. 49, Москва, Россия, 127550
}

Сверхновые типа Іа используются как стандартные свечи в современной космологии, служат для проверки космологических моделей. Интерпретация диаграммы Хаббла на основе стандартной космологической модели привела космологов к заключению, что Вселенная заполнена в основном космической пылью и загадочной тёмной энергией.

В настоящей работе представлены точные решения уравнения Фридмана в стандартной космологии и конформной космологии. Теоретические кривые, интерполирующие диаграмму Хаббла для последних данных по сверхновым, выражаются в аналитическом виде. Функции принадлежат классу мероморфных функций Вейерштрасса. Оба подхода описывают современную диаграмму Хаббла с одинаковой точностью. Физическая интерпретация с позиций конформной космологии предпочтительнее, поскольку данные по сверхновым описываются без использования $\Lambda$-члена. В стандартной космологии для описания диаграммы Хаббла введены характеристики: параметры Хаббла $H(t)$, замедления $q(t)$ и толчка $j(t)$. Как показывают расчёты, параметр замедления $q$ меняет свой знак в течение эволюции Вселенной, $j$-параметр остаётся постоянным. В современную эпоху Вселенная расширяется с ускорением, а в прошлом её ускорение было отрицательным. Изменение знака ускорения, без ясной физической причины, озадачивает космологов.

Нам представляется очевидным, что для исследования объектов, находящихся от нас на расстояниях миллиардов световых лет, следует пользоваться не координатным временем, привычным для работы в лабораториях, а конформным временем. В конформных координатах поведение фотонов описывается как в пространстве Минковского. Интервалы времени $\mathrm{d} t$ и $\mathrm{d} \eta$ отличаются, их связывает масштабный фактор: $\mathrm{d} t=a \mathrm{~d} \eta$. Конформное фотометрическое расстояние длиннее стандартного фотометрического расстояния, что проявляется при наблюдении за удалёнными звёздными объектами. В результате соотношения: эффективная звёздная величина - красное смещение, на которых строится диаграмма Хаббла, будут разными. Используя конформное уравнение Фридмана, в работе вводятся конформные параметры $H(\eta), q(\eta), j(\eta)$. Все параметры остаются положительными в течение эволюции Вселенной. Масштабный фактор растёт с замедлением. Вселенная не испытывает толчка.

Ключевые слова: сверхновые типа Іа, уравнение Фридмана, функции Вейерштрасса, диаграмма Хаббла, космологические параметры, $\Lambda$-член

\section{Литература}

1. Riess A. G. et al. The Farthest Known Supernova: Support for an Accelerating Universe and a Glimpse of the Epoch of Deceleration // The Astrophysical Journal. 2001. - Vol. 560. - Pp. 49-71.

2. Riess A. G. et al. Type Ia Supernova Discoveries at $z>1$ from the Hubble Space Telescope: Evidence for Past Deceleration and Constraints on Dark Energy Evolution // The Astrophysical Journal. — 2004. — Vol. 607. — Pp. 665-687.

3. Riess A. G. Nobel Lecture: My Path to the Accelerating Universe // Reviews of Modern Physics. — 2012. — Vol. 84. - Pp. 1165-1175.

4. Pervushin V. N., Pavlov A. E. Principles of Quantum Universe. - Saarbrücken: Lambert Academic Publishing, 2014. — 480 p. 
5. Dirac P. A. M. Fixation of Coordinates in the Hamiltonian Theory of Gravitation // Physical Review. - 1959. - Vol. 114. — Pp. 924-930.

6. Behnke D. et al. Description of Supernova Data in Conformal Cosmology without Cosmological Constant // Physics Letters. - 2002. - Vol. 30 B. - Pp. 20-26.

7. Behnke D. Conformal cosmology approach to the problem of dark matter: Ph.D. thesis / PhD Thesis, Rostock Report MPG-VT-UR 248/04. - 2004. - 155 p.

8. Zakharov A. F., Pervushin V. N., Zinchuk V. A. Tetrad Formulation and Frames of Reference in General Relativity // Physics of Particles and Nuclei. — 2006. Vol. 37. - Pp. 104-134.

9. Zakharov A. F., Pervushin V. N. Conformal Cosmological Model Parameters with Distant SNe Ia Data: "Gold" and "Silver" // International Journal of Modern Physics. - 2010. — Vol. 19 D. - Pp. 1875-1886.

10. Уиттекер Э. Т., Ватсон Д. Н. Курс современного анализа. - Москва: ГИФМЛ, 1963. - T. 2, 500 c.

11. Pavlov A. E. Exact Solutions of Friedmann Equation for Supernovae Data. 2015. - https://arxiv.org/pdf/1511.00226.pdf.

12. Pavlov A. E. Intrinsic Time in Geometrodynamics: Introduction and Application to Friedmann Cosmology. - 2016. — https://arxiv.org/pdf/1606.09460.

13. Pavlov A. E. Parameters of the Hubble Diagram // 2-nd International Winter SchoolSeminar on gravity, cosmology, and astrophysics. "Petrov School - 2016", Kazan Federal University. - 2016. - Pp. 29-30.

14. Вайнберг С. Космология. - Москва: УРСС, 2013. - 608 с.

15. Zel'dovich Ya. B. The Equation of State at Ultrahigh Densities and its Relativistic Limitations // Soviet Physics JETP. - 1962. - Vol. 14. - Pp. 1143-1147.

16. Нарликар Д. Неистовая Вселенная. - Москва: Мир, 1985. - 256 с.

17. Ахиезер Н. И. Лекции по теории аппроксимации. - Москва: Наука, 1965. $408 \mathrm{c}$.

18. Ахиезер Н. И. Элементы теории эллиптических функций. - Москва: Наука, 1970. - 304 c.

(C) Pavlov A.E., 2017

Для цитирования:

Pavlov A. E. Two Approaches to Interpretation of Hubble Diagram // RUDN Journal of Mathematics, Information Sciences and Physics. - 2017. — Vol. 25, No 4. - Pp. 390400. - DOI: $10.22363 / 2312-9735-2017-25-4-390-400$.

For citation:

Pavlov A.E. Two Approaches to Interpretation of Hubble Diagram, RUDN Journal of Mathematics, Information Sciences and Physics 25 (4) (2017) 390-400. DOI: 10.22363/2312-9735-2017-25-4-390-400.

Сведения об авторах:

Павлов Александр Егорович - доцент, кандидат физико-математических наук, доцент кафедры сопротивления материалов и деталей машин Российского государственного аграрного университета - MCXА имени К.А. Тимирязева (e-mail: alexpavlov60@mail.ru)

Information about the authors:

Pavlov A. E. - Candidate of Physical and Mathematical Sciences, associate professor of Department of Strength of Materials and Machine Parts of Russian State Agrarian University - Moscow Timiryazev Agricultural Academy (e-mail: alexpavlov60@mail.ru) 